\title{
A Novel Characteristic Expanded Mixed Method for Reaction-Convection-Diffusion Problems
}

\author{
Yang Liu, Hong Li, Wei Gao, Siriguleng He, and Zhichao Fang \\ School of Mathematical Sciences, Inner Mongolia University, Hohhot 010021, China \\ Correspondence should be addressed to Yang Liu; mathliuyang@yahoo.cn and Hong Li; smslh@imu.edu.cn
}

Received 3 November 2012; Revised 24 February 2013; Accepted 10 March 2013

Academic Editor: Alexander Timokha

Copyright (C) 2013 Yang Liu et al. This is an open access article distributed under the Creative Commons Attribution License, which permits unrestricted use, distribution, and reproduction in any medium, provided the original work is properly cited.

A novel characteristic expanded mixed finite element method is proposed and analyzed for reaction-convection-diffusion problems. The diffusion term $\nabla \cdot(a(\mathbf{x}, t) \nabla u)$ is discretized by the novel expanded mixed method, whose gradient belongs to the square integrable space instead of the classical $\mathbf{H}(\operatorname{div} ; \Omega)$ space and the hyperbolic part $d(\mathbf{x})(\partial u / \partial t)+\mathbf{c}(\mathbf{x}, t) \cdot \nabla u$ is handled by the characteristic method. For a priori error estimates, some important lemmas based on the novel expanded mixed projection are introduced. The fully discrete error estimates based on backward Euler scheme are obtained. Moreover, the optimal a priori error estimates in $L^{2}$ - and $H^{1}$-norms for the scalar unknown $u$ and a priori error estimates in $\left(L^{2}\right)^{2}$-norm for its gradient $\lambda$ and its flux $\sigma$ (the coefficients times the negative gradient) are derived. Finally, a numerical example is provided to verify our theoretical results.

\section{Introduction}

In this paper, we consider the following reaction-convectiondiffusion problems:

$$
\begin{gathered}
d(\mathbf{x}) \frac{\partial u}{\partial t}+\mathbf{c}(\mathbf{x}, t) \cdot \nabla u-\nabla \cdot(a(\mathbf{x}, t) \nabla u)+R(\mathbf{x}, t) u \\
=f(\mathbf{x}, t), \quad(\mathbf{x}, t) \in \Omega \times J, \\
u(\mathbf{x}, t)=0, \quad(\mathbf{x}, t) \in \partial \Omega \times \bar{J}, \\
u(\mathbf{x}, 0)=u_{0}(\mathbf{x}), \quad \mathbf{x} \in \Omega,
\end{gathered}
$$

where $\Omega$ is a bounded convex polygonal domain in $\mathbb{R}^{2}$ with Lipschitz continuous boundary $\partial \Omega$ and $J=(0, T]$ is the time interval with $0<T<\infty$. The initial value $u_{0}(\mathbf{x})$ and the source term $f(\mathbf{x}, t)$ are given functions. Throughout this paper, we assume that the accumulation, diffusion, and reaction coefficients, $d=d(\mathbf{x}), a=a(\mathbf{x}, t)$, and $R=R(\mathbf{x}, t)$, satisfy

$$
\begin{gathered}
\mathbf{H}_{1}: 0<d_{0} \leq d(\mathbf{x}) \leq d_{1}<+\infty, \\
\mathbf{H}_{2}: 0<a_{0} \leq a(\mathbf{x}, t) \leq a_{1}<+\infty, \quad\left|a_{t}(\mathbf{x}, t)\right| \leq \bar{a}_{1}, \\
\mathbf{H}_{3}: 0 \leq R_{0} \leq R(\mathbf{x}, t) \leq R_{1}<+\infty, \quad\left|R_{t}(\mathbf{x}, t)\right| \leq \bar{R}_{1},
\end{gathered}
$$

and the bounded vector $\mathbf{c}(\mathbf{x}, t)=\left(c_{1}(\mathbf{x}, t), c_{2}(\mathbf{x}, t)\right)$ satisfies

$$
\mathbf{H}_{4}: 0<b_{0} \leq\left(\sum_{i=1}^{2} c_{i}^{2}(\mathbf{x}, t)\right)^{1 / 2} \leq b_{1}<+\infty .
$$

Reaction-convection-diffusion equations are a class of important evolution partial differential equations and have a lot of applications in many physical problems, such as the infiltration of liquid, the proliferation of gas, the conduction of heat, and the spread of impurities in semiconductor materials. In recent years, a lot of numerical methods, such as mixed finite element methods [1-3], Pod methods $[4,5]$, characteristic-mixed covolume methods [6], spacetime discontinuous Galerkin methods $[7,8]$, least-squares finite element methods $[9,10]$, nonconforming finite element method [11], conforming rectangular element method [12], and two-grid expanded mixed methods [13-16], have been studied for reaction-convection-diffusion equations.

In 1994, Chen $[17,18]$ proposed an expanded mixed finite element method for second-order linear elliptic equation. Compared to standard mixed element methods, the expanded mixed method can approximate three variables simultaneously, namely, the scalar unknown, its gradient, and its flux (the tensor coefficient times the negative gradient). From then on, the expanded mixed method was applied 
to many evolution equations $[2,19,20]$, and some new numerical methods based on the Chen's expanded mixed method were proposed, for example, expanded mixed covolume method [21], expanded mixed hybrid methods [22], positive definite expanded mixed method [23], and so on. From the above literature on the study of expanded mixed method, we can find that all papers were studied based on Chen's expanded mixed method $[17,18]$.

In 2011, we developed a new expanded mixed finite element method [24] based on the mixed weak formulations [25-27]. In this paper, we develop and analyze a novel characteristic expanded mixed finite element method, which combines the novel expanded mixed method [24] applied to approximating the diffusion term and the characteristic method that handled the hyperbolic part, for reactionconvection-diffusion equations. The gradient for our method belongs to the square integrable space instead of the classical $\mathbf{H}(\operatorname{div} ; \Omega)$ space of Chen's expanded mixed method. We derive a priori error estimates based on backward Euler method. Moreover, we prove the optimal a priori error estimates in $L^{2}$ - and $H^{1}$-norms for the scalar unknown $u$ and a priori error estimates in $\left(L^{2}\right)^{2}$-norm for its gradient $\lambda$ and its flux $\sigma$ (the coefficients times the negative gradient).

Throughout this paper, $C$ will denote a generic positive constant which does not depend on the spatial mesh parameter $h$ and time discretization parameter $\Delta t$. At the same time, we denote the natural inner product in $L^{2}(\Omega)$ or $\left(L^{2}(\Omega)\right)^{2}$ by $(\cdot, \cdot)$ with the corresponding norm $\|\cdot\|$. The other notations and definitions of Sobolev spaces as in [28] are used.

\section{Novel Characteristic Expanded Mixed System}

Introducing the two auxiliary variables $\lambda=\nabla u, \sigma=$ $-(a(\mathbf{x}, t) \nabla u)=-(a(\mathbf{x}, t) \lambda)$, we obtain the following first-order system for (1):

$$
\begin{gathered}
d \frac{\partial u}{\partial t}+\mathbf{c} \cdot \nabla u+\nabla \cdot \sigma+R u=f(\mathbf{x}, t) \\
\lambda-\nabla u=0 \\
\sigma+a \lambda=0 .
\end{gathered}
$$

Let the characteristic direction corresponding to the hyperbolic part $d \partial u / \partial t+\mathbf{c} \cdot \nabla u$ be denoted by $\tau=\tau(\mathbf{x})$, which is subject to

$$
\frac{\partial}{\partial \tau(\mathbf{x})}=\frac{d(\mathbf{x})}{\psi(\mathbf{x}, t)} \frac{\partial}{\partial t}+\frac{\mathbf{c}(\mathbf{x})}{\psi(\mathbf{x}, t)} \cdot \nabla
$$

where $\psi(\mathbf{x}, t)=\left(d^{2}(\mathbf{x})+|\mathbf{c}(\mathbf{x}, t)|^{2}\right)^{1 / 2}$.

Then the first-order system (4) can be rewritten as

$$
\begin{gathered}
\psi \frac{\partial u}{\partial \tau}+\nabla \cdot \sigma+R u=f(\mathbf{x}, t), \\
\lambda-\nabla u=0 \\
\sigma+a \lambda=0
\end{gathered}
$$

Using the similar method to the one in [29], the expanded mixed weak formulation for problem (1) is to find $\{u, \lambda, \sigma\}$ : $[0, T] \mapsto W \times \mathbf{V} \times \mathbf{X}$ such that

$$
\begin{gathered}
\left(\psi \frac{\partial u}{\partial \tau}, v\right)+(\nabla \cdot \sigma, v)+(R u, v)=(f, v), \quad \forall v \in W \\
(\lambda, \mathbf{w})+(u, \nabla \cdot \mathbf{w})=0, \quad \forall \mathbf{w} \in \mathbf{V} \\
(\sigma, \mathbf{z})+(a \lambda, \mathbf{z})=0, \quad \forall \mathbf{z} \in \mathbf{X}
\end{gathered}
$$

where $W=L^{2}(\Omega)$ or $W=\left\{w \in L^{2}(\Omega)|w|_{\partial \Omega}=0\right\}, \mathbf{V}=$ $\mathbf{H}(\operatorname{div} ; \Omega)=\left\{\mathbf{v} \in\left(L^{2}(\Omega)\right)^{2} \mid \nabla \cdot \mathbf{v} \in L^{2}(\Omega)\right\}$, and $\mathbf{X}=\left(L^{2}(\Omega)\right)^{2}$.

In this paper, we propose and discuss a novel characteristic expanded mixed method. The new characteristic expanded mixed weak formulation is to find $\{u, \lambda, \sigma\}$ : $[0, T] \mapsto H_{0}^{1} \times\left(L^{2}(\Omega)\right)^{2} \times\left(L^{2}(\Omega)\right)^{2}$ such that

$$
\begin{gathered}
\left(\psi \frac{\partial u}{\partial \tau}, v\right)-(\sigma, \nabla v)+(R u, v)=(f, v), \quad \forall v \in H_{0}^{1} \\
(\lambda, \mathbf{w})-(\nabla u, \mathbf{w})=0, \quad \forall \mathbf{w} \in\left(L^{2}(\Omega)\right)^{2}, \\
(\sigma, \mathbf{z})+(a \lambda, \mathbf{z})=0, \quad \forall \mathbf{z} \in\left(L^{2}(\Omega)\right)^{2} .
\end{gathered}
$$

For approximating the solution at time $t_{n}=n \Delta t$, the characteristic derivative will be approximated by

$$
\widehat{\mathbf{x}}_{n}=\mathbf{x}-\frac{\Delta t}{d(\mathbf{x})} c\left(\mathbf{x}, t_{n}\right),
$$

and then we have the following approximation:

$$
\begin{aligned}
\left.\psi\left(\mathbf{x}, t_{n}\right) \frac{\partial u}{\partial \tau}\right|_{t_{n}} & \approx \psi\left(\mathbf{x}, t_{n}\right) \frac{u\left(\mathbf{x}, t_{n}\right)-u\left(\widehat{\mathbf{x}}_{n}, t_{n-1}\right)}{\sqrt{\left(\mathbf{x}-\widehat{\mathbf{x}}_{n}\right)^{2}+(\Delta t)^{2}}} \\
& =d(\mathbf{x}) \frac{u\left(\mathbf{x}, t_{n}\right)-u\left(\widehat{\mathbf{x}}_{n}, t_{n-1}\right)}{\Delta t} .
\end{aligned}
$$

Let $\left(V_{h}, \mathbf{W}_{h}\right) \subset\left(H_{0}^{1},\left(L^{2}(\Omega)\right)^{2}\right)$ be defined by the following finite element pair $P_{1}-P_{0}^{2}[25,26]$ :

$$
V_{h}=\left\{v_{h} \in C^{0}(\Omega) \cap H_{0}^{1} \mid v_{h} \in P_{1}(K), \forall K \in \mathscr{K}_{h}\right\}
$$

$$
\begin{aligned}
& \mathbf{W}_{h} \\
& =\left\{\mathbf{w}_{h}=\left(w_{1 h}, w_{2 h}\right) \in\left(L^{2}(\Omega)\right)^{2} \mid w_{1 h}, w_{2 h} \in P_{0}(K),\right. \\
& \left.\forall K \in \mathscr{K}_{h}\right\} .
\end{aligned}
$$


Now the novel characteristic expanded mixed finite element procedure for (8a), (8b), and (8c) is to find $\left\{u_{h}^{n}, \lambda_{h}^{n}, \sigma_{h}^{n}\right\}$ : $[0, T] \mapsto V_{h} \times \mathbf{W}_{h} \times \mathbf{W}_{h}$ satisfying

$$
\begin{aligned}
& \left(d \frac{u_{h}^{n}-\widehat{u}_{h}^{n-1}}{\Delta t}, v_{h}\right)-\left(\sigma_{h}^{n}, \nabla v_{h}\right)+\left(R u_{h}^{n}, v_{h}\right) \\
& =\left(f^{n}, v_{h}\right), \quad \forall v_{h} \in V_{h}, \\
& \left(\lambda_{h}^{n}, \mathbf{w}_{h}\right)-\left(\nabla u_{h}^{n}, \mathbf{w}_{h}\right)=0, \quad \forall \mathbf{w}_{h} \in \mathbf{W}_{h}, \\
& \left(\sigma_{h}^{n}, \mathbf{z}_{h}\right)+\left(a^{n} \lambda_{h}^{n}, \mathbf{z}_{h}\right)=0, \quad \forall \mathbf{z}_{h} \in \mathbf{W}_{h},
\end{aligned}
$$

where $u_{h}^{n}=u_{h}\left(t_{n}\right), \widehat{u}_{h}^{n-1}=u_{h}\left(\widehat{\mathbf{x}}_{n}, t_{n-1}\right)=u_{h}(\mathbf{x}-$ $\left.(\Delta t / d(\mathbf{x})) c\left(\mathbf{x}, t_{n}\right), t_{n-1}\right)$.

Remark 1. From $[25,26]$, we find that $\left(V_{h}, \mathbf{W}_{h}\right)$ satisfies the so-called discrete Ladyzhenskaya-Babuska-Brezzi condition.

Remark 2. Compared to the scheme (7a), (7b), and (7c) based on Chen's expanded mixed element method, the gradient in the scheme (8a), (8b), and (8c) belongs to the simple square integrable space instead of the classical $\mathbf{H}(\operatorname{div} ; \Omega)$ space. For $\mathbf{H}(\operatorname{div} ; \Omega) \subset\left(L^{2}(\Omega)\right)^{2}$, we easily find that our method reduces the regularity requirement on the gradient solution $\lambda=\nabla u$.

Remark 3. Based on finite element space $\left(V_{h}, \mathbf{W}_{h}\right)$ in (11), the number of total degrees of freedom for our scheme ((12a), (12b), and (12c)) is less than that for the scheme in [29]. By the same discussion as Remark 1 in [30], we can obtain the detailed analysis for degrees of freedom.

\section{Some Lemmas and Error Estimates}

3.1. Novel Expanded Mixed Projection and Lemmas. We first introduce the novel expanded mixed elliptic projection [24] associated with our equations to derive a priori error estimates for the proposed method.

Let $\left(\tilde{u}_{h}, \tilde{\lambda}_{h}, \tilde{\sigma}_{h}\right):[0, T] \rightarrow V_{h} \times \mathbf{W}_{h} \times \mathbf{W}_{h}$ be given by the following mixed relations:

$$
\begin{gathered}
\left(\sigma-\widetilde{\sigma}_{h}, \nabla v_{h}\right)=0, \quad \forall v_{h} \in V_{h}, \\
\left(\lambda-\widetilde{\lambda}_{h}, \mathbf{w}_{h}\right)-\left(\nabla\left(u-\widetilde{u}_{h}\right), \mathbf{w}_{h}\right)=0, \quad \forall \mathbf{w}_{h} \in \mathbf{W}_{h}, \\
\left(\sigma-\widetilde{\sigma}_{h}, \mathbf{z}_{h}\right)+\left(a\left(\lambda-\tilde{\lambda}_{h}\right), \mathbf{z}_{h}\right)=0, \quad \forall \mathbf{z}_{h} \in \mathbf{W}_{h} .
\end{gathered}
$$

In the following discussion, we will give some important lemmas based on the novel expanded mixed projection.

Lemma 4 (see [24]). There is a constant $C$ independent of $h$ such that

$$
\begin{gathered}
\|\delta\| \leq \operatorname{Ch}\left(\|\lambda\|_{\left(H^{1}(\Omega)\right)^{2}}+\|u\|_{H^{2}}\right), \\
\|\rho\| \leq \operatorname{Ch}\left(\|u\|_{H^{2}}+\|\sigma\|_{\left(H^{1}(\Omega)\right)^{2}}+\|\lambda\|_{\left(H^{1}(\Omega)\right)^{2}}\right), \\
\|\nabla \eta\| \leq \operatorname{Ch}\left(\|u\|_{H^{2}}+\|\lambda\|_{\left(H^{1}(\Omega)\right)^{2}}\right),
\end{gathered}
$$

where $\eta=u-\tilde{u}_{h}, \delta=\lambda-\tilde{\lambda}_{h}$, and $\rho=\sigma-\widetilde{\sigma}_{h}$.
Lemma 5 (see [24]). There is a constant $C$ independent of $h$ such that

$$
\begin{gathered}
\|\eta\| \leq C h^{2}\|u\|_{H^{2}}, \\
\left\|\eta_{t}\right\| \leq C h^{2}\left\|u_{t}\right\|_{H^{2}}, \\
\|\eta\|_{1} \leq C h\left(\|u\|_{H^{2}}+\|\lambda\|_{\left(H^{1}(\Omega)\right)^{2}}\right) .
\end{gathered}
$$

Lemma 6 (see [6]). For each $n$ one has

$$
(d \widehat{v}, \widehat{v})-(d v, v) \leq C \Delta t(d v, v), \quad \forall v \in L^{2}(\Omega),
$$

where $\widehat{v}=v\left(\mathbf{x}-\mathbf{c}\left(\mathbf{x}, t_{n}\right) \Delta t / d(\mathbf{x})\right)$.

Remark 7. For proving Lemmas 4 and 5, we introduce two linear operators $[25,26] \Pi_{h}:\left(L^{2}(\Omega)\right)^{2} \rightarrow \mathbf{W}_{h}$ and $P_{h}:$ $H_{0}^{1}(\Omega) \rightarrow V_{h}$ and consider the following auxiliary elliptic problem:

$$
\begin{gathered}
-\nabla \cdot(a \nabla \chi)=\eta, \quad \text { in } \Omega, \\
\chi=0, \quad \text { on } \partial \Omega .
\end{gathered}
$$

The detailed proofs for Lemmas 4 and 5 are shown in [24].

3.2. A Priori Error Estimates. In the following discussion, we will derive a priori error estimates based on fully discrete backward Euler method. Let $0=t_{0}<t_{1}<t_{2}<\cdots<t_{M}=T$ be a given partition of the time interval $[0, T]$ with step length $\Delta t=T / M$ and nodes $t_{n}=n \Delta t$, for some positive integer $M$. For a smooth function $\phi$ on $[0, T]$, define $\phi^{n}=\phi\left(t_{n}\right)$.

In order to derive a priori error estimates, we now write

$$
\begin{aligned}
& u\left(t_{n}\right)-u_{h}^{n}=\left(u\left(t_{n}\right)-\widetilde{u}_{h}^{n}\right)+\left(\widetilde{u}_{h}^{n}-u_{h}^{n}\right)=\eta^{n}+\varsigma^{n}, \\
& \lambda\left(t_{n}\right)-\lambda_{h}^{n}=\left(\lambda\left(t_{n}\right)-\tilde{\lambda}_{h}^{n}\right)+\left(\tilde{\lambda}_{h}^{n}-\lambda_{h}^{n}\right)=\delta^{n}+\theta^{n}, \\
& \sigma\left(t_{n}\right)-\sigma_{h}^{n}=\left(\sigma\left(t_{n}\right)-\widetilde{\sigma}_{h}^{n}\right)+\left(\widetilde{\sigma}_{h}^{n}-\sigma_{h}^{n}\right)=\rho^{n}+\xi^{n} .
\end{aligned}
$$

Combining (8a), (8b), and (8c), (12a), (12b), and (12c) and (13a), (13b), and (13c) at $t=t_{n}$, we get the error equations

$$
\begin{aligned}
& \left(d \frac{\varsigma^{n}-\widehat{\varsigma}^{n-1}}{\Delta t}, v_{h}\right)-\left(\xi^{n}, \nabla v_{h}\right)+\left(R \varsigma^{n}, v_{h}\right) \\
& =\left(\psi^{n} \frac{\partial u^{n}}{\partial \tau}-d \frac{u^{n}-\widehat{u}^{n-1}}{\Delta t}, v_{h}\right) \\
& \quad-\left(d \frac{\eta^{n}-\widehat{\eta}^{n-1}}{\Delta t}, v_{h}\right)-\left(R \eta^{n}, v_{h}\right), \quad \forall v_{h} \in V_{h} \\
& \left(\theta^{n}, \mathbf{w}_{h}\right)-\left(\nabla \varsigma^{n}, \mathbf{w}_{h}\right)=0, \quad \forall \mathbf{w}_{h} \in \mathbf{W}_{h}, \\
& \left(\xi^{n}, \mathbf{z}_{h}\right)+\left(a^{n} \theta^{n}, \mathbf{z}_{h}\right)=0, \quad \forall \mathbf{z}_{h} \in \mathbf{W}_{h} .
\end{aligned}
$$

Based on the error equations (21a), (21b), and (21c), we obtain the following theorem for the fully discrete error estimates. 
Theorem 8. Assume that $u_{h}^{0}=\widetilde{u}_{h}(0)$; then there exist some positive constants independent of $h=O(\Delta t)$ such that

$$
\begin{aligned}
\left\|u\left(t_{J}\right)-u_{h}^{J}\right\| \leq & C\left(a_{0}, d_{1}\right) \mid\left\|O\left(h^{2}, \Delta t\right)\right\| \| \\
& +C\left(a_{0}, d_{1}, R_{1}\right) h^{2}\|u\|_{L^{\infty}\left(H^{2}\right)}, \\
\left\|u\left(t_{J}\right)-u_{h}^{J}\right\|_{1} \leq & C\left(a_{0}, d_{1}\right)\left\|O O\left(h^{1}, \Delta t\right)\right\| \| \\
+ & h\left[C\left(a_{0}, d_{1}, R_{1}\right)\|u\|_{L^{\infty}\left(H^{2}\right)}\right. \\
\left.+C\|\lambda\|_{L^{\infty}\left(\left(H^{1}\right)^{2}\right)}\right], & \\
\left\|\lambda\left(t_{J}\right)-\lambda_{h}^{J}\right\| \leq & C\left(a_{0}, d_{1}, R_{1}\right)\left\|O\left(h^{1}, \Delta t\right)\right\| \\
+ & h\left[C\left(a_{0}, d_{1}, R_{1}, \bar{R}_{1}\right)\|u\|_{L^{\infty}\left(H^{2}\right)}\right. \\
& \left.+C\|\lambda\|_{L^{\infty}\left(\left(H^{1}\right)^{2}\right)}\right], \\
\left\|\sigma\left(t_{J}\right)-\sigma_{h}^{J}\right\| \leq & C\left(a_{0}, d_{1}, R_{1}\right)\left\|O\left(h^{1}, \Delta t\right)\right\| \| \\
+ & h\left[C\left(\|\lambda\|_{L^{\infty}\left(\left(H^{1}\right)^{2}\right)}+\|\sigma\|_{L^{\infty}\left(\left(H^{1}\right)^{2}\right)}\right)\right. \\
& \left.+C\left(a_{0}, d_{1}, R_{1}, \bar{R}_{1}\right)\|u\|_{L^{\infty}\left(H^{2}\right)}\right],
\end{aligned}
$$

where \|\|$O\left(h^{2-j}, \Delta t\right) \| \mid=h^{2-j}\left(\|u\|_{L^{\infty}\left(H^{2}\right)}+\left\|u_{t}\right\|_{L^{2}\left(H^{2}\right)}\right)+$ $\Delta t|| u_{t t} \|_{L^{2}\left(L^{2}\right)}, j=0,1$.

Proof. Set $v_{h}=\varsigma^{n}$ in (21a), $\mathbf{w}_{h}=\xi^{n}$ in (21b), and $\mathbf{z}_{h}=\theta^{n}$ in (21c) to obtain

$$
\begin{gathered}
\left(d \frac{\varsigma^{n}-\widehat{\varsigma}^{n-1}}{\Delta t}, \varsigma^{n}\right)-\left(\xi^{n}, \nabla \varsigma^{n}\right)+\left(R^{n} \varsigma^{n}, \varsigma^{n}\right) \\
=\left(\psi^{n} \frac{\partial u^{n}}{\partial \tau}-d \frac{u^{n}-\widehat{u}^{n-1}}{\Delta t}, \varsigma^{n}\right) \\
-\left(d \frac{\eta^{n}-\widehat{\eta}^{n-1}}{\Delta t}, \varsigma^{n}\right)-\left(R^{n} \eta^{n}, \varsigma^{n}\right), \\
\left(\nabla \varsigma^{n}, \xi^{n}\right)-\left(\theta^{n}, \xi^{n}\right)=0, \\
\left(\xi^{n}, \theta^{n}\right)+\left\|\left(a^{n}\right)^{1 / 2} \theta^{n}\right\|^{2}=0 .
\end{gathered}
$$

Adding the above three equations, we obtain

$$
\begin{gathered}
\left(d \frac{\varsigma^{n}-\widehat{\varsigma}^{n-1}}{\Delta t}, \varsigma^{n}\right)+\left\|\left(a^{n}\right)^{1 / 2} \theta^{n}\right\|^{2}+\left\|\left(R^{n}\right)^{1 / 2} \varsigma^{n}\right\|^{2} \\
=\left(\psi^{n} \frac{\partial u^{n}}{\partial \tau}-d \frac{u^{n}-\widehat{u}^{n-1}}{\Delta t}, \varsigma^{n}\right) \\
-\left(d \frac{\eta^{n}-\hat{\eta}^{n-1}}{\Delta t}, \varsigma^{n}\right)-\left(R^{n} \eta^{n}, \varsigma^{n}\right) .
\end{gathered}
$$

Noting that $-d \widehat{\varsigma}^{n-1} \varsigma^{n} / \Delta t=\left(\left\|d^{1 / 2}\left(\varsigma^{n}-\widehat{\varsigma}^{n-1}\right)\right\|^{2}-\left(\left\|d^{1 / 2} \widehat{\varsigma}^{n-1}\right\|^{2}+\right.\right.$ $\left.\left.\left\|d^{1 / 2} \varsigma^{n}\right\|^{2}\right)\right) / 2 \Delta t$, (24) may be written as

$$
\begin{aligned}
\frac{\left\|d^{1 / 2} \varsigma^{n}\right\|^{2}-\left\|d^{1 / 2} \widehat{\varsigma}^{n-1}\right\|^{2}}{2 \Delta t}+a_{0}\left\|\theta^{n}\right\|^{2}+\left\|\left(R^{n}\right)^{1 / 2} \varsigma^{n}\right\|^{2} \\
\leq \frac{\left\|d^{1 / 2} \varsigma^{n}\right\|^{2}-\left\|d^{1 / 2} \widehat{\varsigma}^{n-1}\right\|^{2}+\left\|d^{1 / 2}\left(\varsigma^{n}-\widehat{\varsigma}^{n-1}\right)\right\|^{2}}{2 \Delta t} \\
\quad+\left\|\left(a^{n}\right)^{1 / 2} \theta^{n}\right\|^{2}+\left\|\left(R^{n}\right)^{1 / 2} \varsigma^{n}\right\|^{2} \\
=\left(\psi^{n} \frac{\partial u^{n}}{\partial \tau}-d \frac{u^{n}-\widehat{u}^{n-1}}{\Delta t}, \varsigma^{n}\right) \\
\\
\quad-\left(d \frac{\eta^{n}-\widehat{\eta}^{n-1}}{\Delta t}, \varsigma^{n}\right)-\left(R^{n} \eta^{n}, \varsigma^{n}\right) .
\end{aligned}
$$

Using the same analysis as that in [31], the right-hand side of (25) can be rewritten as

$$
\begin{aligned}
\left(\psi^{n}\right. & \left.\frac{\partial u^{n}}{\partial \tau}-d \frac{u^{n}-\widehat{u}^{n-1}}{\Delta t}, \varsigma^{n}\right)-\left(d \frac{\eta^{n}-\widehat{\eta}^{n-1}}{\Delta t}, \varsigma^{n}\right)-\left(R^{n} \eta^{n}, \varsigma^{n}\right) \\
= & \left(\psi^{n} \frac{\partial u^{n}}{\partial \tau}-d \frac{u^{n}-\widehat{u}^{n-1}}{\Delta t}, \varsigma^{n}\right) \\
& -\left(d \frac{\eta^{n}-\eta^{n-1}+\eta^{n-1}-\widehat{\eta}^{n-1}}{\Delta t}, \varsigma^{n}\right)-\left(R^{n} \eta^{n}, \varsigma^{n}\right) \\
\leq & \left(\left\|\psi^{n} \frac{\partial u^{n}}{\partial \tau}-d \frac{u^{n}-\widehat{u}^{n-1}}{\Delta t}\right\|+\left\|d \frac{\eta^{n}-\eta^{n-1}}{\Delta t}\right\|\right. \\
& \left.+\left\|d \frac{\eta^{n-1}-\hat{\eta}^{n-1}}{\Delta t}\right\|+\left\|R^{n} \eta^{n}\right\|\right)\left\|\varsigma^{n}\right\| \\
\leq & C\left(d_{1}\right)\left[\Delta t \int_{t_{n-1}}^{t_{n}}\left\|\frac{\partial^{2} u}{\partial \tau^{2}}\right\|^{2} d s+\frac{1}{\Delta t} \int_{t_{n-1}}^{t_{n}}\left\|\frac{\partial \eta}{\partial \tau}\right\|^{2} d s+\left\|\varsigma^{n}\right\|^{2}\right] \\
& +\left\|R^{n} \eta^{n}\right\|^{2}+\left\|d \frac{\eta^{n-1}-\widehat{\eta}^{n-1}}{\Delta t}\right\|\left\|\varsigma^{n}\right\| .
\end{aligned}
$$

Using the assumption $h=O(\Delta t)$, we obtain

$$
\begin{aligned}
\left\|d \frac{\eta^{n-1}-\hat{\eta}^{n-1}}{\Delta t}\right\|\left\|\varsigma^{n}\right\| & \leq C\left(\frac{h+\Delta t}{\Delta t}\right)\left\|\eta^{n-1}\right\|\left\|\theta^{n}\right\| \\
& \leq C\left(a_{0}\right)\|\eta\|_{L^{\infty}\left(L^{2}\right)}^{2}+\frac{a_{0}}{2}\|\theta\|^{2} .
\end{aligned}
$$

By Lemma 6, we have

$$
\left\|d^{1 / 2} \varsigma^{n-1}\right\|^{2}-\left\|d^{1 / 2 \varkappa^{n-1}}\right\|^{2} \geq-C \Delta t\left\|d^{1 / 2} \varsigma^{n-1}\right\|^{2}
$$


Using (28), we obtain

$$
\begin{gathered}
\frac{\left\|d^{1 / 2} \varsigma^{n}\right\|^{2}-\left\|d^{1 / 2} \widehat{\varsigma}^{n-1}\right\|^{2}}{2 \Delta t}+a_{0}\left\|\theta^{n}\right\|^{2}+\left\|\left(R^{n}\right)^{1 / 2} \varsigma^{n}\right\|^{2} \\
\geq \frac{\left\|d^{1 / 2} \varsigma^{n}\right\|^{2}-(1+C \Delta t)\left\|d^{1 / 2} \varsigma^{n-1}\right\|^{2}}{2 \Delta t} \\
+a_{0}\left\|\theta^{n}\right\|^{2}+\left\|\left(R^{n}\right)^{1 / 2} \varsigma^{n}\right\|^{2} \\
=\frac{\left\|d^{1 / 2} \varsigma^{n}\right\|^{2}-\left\|d^{1 / 2} \varsigma^{n-1}\right\|^{2}-C \Delta t\left\|d^{1 / 2} \varsigma^{n-1}\right\|^{2}}{2 \Delta t} \\
+a_{0}\left\|\theta^{n}\right\|^{2}+\left\|\left(R^{n}\right)^{1 / 2} \varsigma^{n}\right\|^{2} .
\end{gathered}
$$

Multiplying by $2 \Delta t$, summing (29) from $n=1$ to $J$, and using (25)-(29), the resulting inequality becomes

$$
\begin{aligned}
d_{0}\left\|\varsigma^{J}\right\|^{2}+\Delta t \sum_{n=1}^{J}\left(a_{0}\left\|\theta^{n}\right\|^{2}+\left\|\left(R^{n}\right)^{1 / 2} \varsigma^{n}\right\|^{2}\right) \\
\leq d_{1}\left\|\varsigma^{0}\right\|^{2}+C\left(a_{0}, d_{1}\right) \\
\times\left[\Delta t \sum_{n=1}^{J}\left(\Delta t \int_{t_{n-1}}^{t_{n}}\left\|\frac{\partial^{2} u}{\partial \tau^{2}}\right\|^{2} d s+\frac{1}{\Delta t} \int_{t_{n-1}}^{t_{n}}\left\|\frac{\partial \eta}{\partial \tau}\right\|^{2} d s\right)\right] \\
+C\left(a_{0}, d_{1}, R_{1}\right)\|\eta\|_{L^{\infty}\left(L^{2}\right)}^{2}+C\left(d_{1}\right) \Delta t \sum_{n=1}^{J}\left\|\varsigma^{n-1}\right\|^{2} \\
=d_{1}\left\|\varsigma^{0}\right\|^{2}+C\left(a_{0}, d_{1}\right) \\
\times\left[(\Delta t)^{2} \int_{t_{0}}^{t_{J}}\left\|\frac{\partial^{2} u}{\partial \tau^{2}}\right\|^{2} d s+\int_{t_{0}}^{t_{J}}\left\|\frac{\partial \eta}{\partial \tau}\right\|^{2} d s\right] \\
+C\left(a_{0}, d_{1}, R_{1}\right)\|\eta\|_{L^{\infty}\left(L^{2}\right)}^{2}+C\left(d_{1}\right) \Delta t \sum_{n=1}^{J}\left\|\varsigma^{n-1}\right\|^{2} .
\end{aligned}
$$

Using Gronwall lemma, we have

$$
\begin{aligned}
d_{0}\left\|\varsigma^{J}\right\|^{2} & +\Delta t \sum_{n=1}^{J}\left(a_{0}\left\|\theta^{n}\right\|^{2}+\left\|\left(R^{n}\right)^{1 / 2} \varsigma^{n}\right\|^{2}\right) \\
\leq & C\left(a_{0}, d_{1}\right)\left[(\Delta t)^{2} \int_{t_{0}}^{t_{J}}\left\|\frac{\partial^{2} u \|^{2}}{\partial \tau^{2}}\right\|^{2} d s+\int_{t_{0}}^{t_{J}}\left\|\frac{\partial \eta}{\partial \tau}\right\|^{2} d s\right] \\
& +C\left(a_{0}, d_{1}, R_{1}\right)\|\eta\|_{L^{\infty}\left(L^{2}\right)}^{2} .
\end{aligned}
$$

From (21b), we get

$$
\left(\frac{\theta^{n}-\theta^{n-1}}{\Delta t}, \mathbf{w}_{h}\right)-\left(\frac{\nabla \varsigma^{n}-\nabla \varsigma^{n-1}}{\Delta t}, \mathbf{w}_{h}\right)=0 .
$$

Choose $\mathbf{w}_{h}=\xi^{n}$ in (32), $v_{h}=\left(\varsigma^{n}-\varsigma^{n-1}\right) / \Delta t$ in (21a), and $\mathbf{z}_{h}=\left(\theta^{n}-\theta^{n-1}\right) / \Delta t$ in (21c) to obtain

$$
\begin{aligned}
& \left(d \frac{\varsigma^{n}-\widehat{\varsigma}^{n-1}}{\Delta t}, \frac{\varsigma^{n}-\varsigma^{n-1}}{\Delta t}\right)-\left(\xi^{n}, \frac{\nabla \varsigma^{n}-\nabla \varsigma^{n-1}}{\Delta t}\right) \\
& \quad+\left(R^{n} \varsigma^{n}, \frac{\varsigma^{n}-\varsigma^{n-1}}{\Delta t}\right) \\
& =\left(\psi^{n} \frac{\partial u^{n}}{\partial \tau}-d \frac{u^{n}-\widehat{u}^{n-1}}{\Delta t}, \frac{\varsigma^{n}-\varsigma^{n-1}}{\Delta t}\right) \\
& \quad-\left(d \frac{\eta^{n}-\hat{\eta}^{n-1}}{\Delta t}, \frac{\varsigma^{n}-\varsigma^{n-1}}{\Delta t}\right)-\left(R^{n} \eta^{n}, \frac{\varsigma^{n}-\varsigma^{n-1}}{\Delta t}\right),
\end{aligned}
$$

$$
\begin{aligned}
& \left(\frac{\nabla \varsigma^{n}-\nabla \varsigma^{n-1}}{\Delta t}, \xi^{n}\right)-\left(\frac{\theta^{n}-\theta^{n-1}}{\Delta t}, \xi^{n}\right)=0, \\
& \left(\xi^{n}, \frac{\theta^{n}-\theta^{n-1}}{\Delta t}\right)+\left(a^{n} \theta^{n}, \frac{\theta^{n}-\theta^{n-1}}{\Delta t}\right)=0 .
\end{aligned}
$$

Adding the three equations and using Cauchy-Schwarz inequality and Young inequality, we obtain

$$
\begin{aligned}
\left(d \frac{\varsigma^{n}-\widehat{\varsigma}^{n-1}}{\Delta t}, \frac{\varsigma^{n}-\varsigma^{n-1}}{\Delta t}\right)+\left(a^{n} \theta^{n}, \frac{\theta^{n}-\theta^{n-1}}{\Delta t}\right) & \\
& +\left(R^{n} \varsigma^{n}, \frac{\varsigma^{n}-\varsigma^{n-1}}{\Delta t}\right) \\
= & \left(\psi^{n} \frac{\partial u^{n}}{\partial \tau}-d \frac{u^{n}-\widehat{u}^{n-1}}{\Delta t}, \frac{\varsigma^{n}-\varsigma^{n-1}}{\Delta t}\right) \\
& -\left(d \frac{\eta^{n}-\widehat{\eta}^{n-1}}{\Delta t}, \frac{\varsigma^{n}-\varsigma^{n-1}}{\Delta t}\right)-\left(R^{n} \eta^{n}, \frac{\varsigma^{n}-\varsigma^{n-1}}{\Delta t}\right) \\
\leq & \left(\left\|\psi^{n} \frac{\partial u^{n}}{\partial \tau}-d \frac{u^{n}-\widehat{u}^{n-1}}{\Delta t}\right\|+\left\|d \frac{\eta^{n}-\eta^{n-1}}{\Delta t}\right\|\right. \\
& \left.+\left\|d \frac{\eta^{n-1}-\widehat{\eta}^{n-1}}{\Delta t}\right\|+\left\|R^{n} \eta^{n}\right\|\right)\left\|\frac{\varsigma^{n}-\varsigma^{n-1}}{\Delta t}\right\| \\
\leq & C\left(d_{1}\right) \Delta t \int_{t_{n-1}}^{t_{n}}\left\|\frac{\partial^{2} u}{\partial \tau^{2}}\right\|^{2} d s+\frac{C\left(d_{1}\right)}{\Delta t} \int_{t_{n-1}}^{t_{n}}\left\|\frac{\partial \eta}{\partial \tau}\right\|^{2} d s \\
& +C\left(R_{1}\right)\|\eta\|_{L^{\infty}\left(L^{2}\right)}^{2}+\frac{1}{2}\left\|\frac{\varsigma^{n}-\varsigma^{n-1}}{\Delta t}\right\|^{2} .
\end{aligned}
$$


The left-hand side of (34) can be written as

$$
\begin{aligned}
(d & \left.\frac{\varsigma^{n}-\widehat{\varsigma}^{n-1}}{\Delta t}, \frac{\varsigma^{n}-\varsigma^{n-1}}{\Delta t}\right)+\left(a^{n} \theta^{n}, \frac{\theta^{n}-\theta^{n-1}}{\Delta t}\right) \\
& +\left(R^{n} \varsigma^{n}, \frac{\varsigma^{n}-\varsigma^{n-1}}{\Delta t}\right) \\
= & \left\|d^{1 / 2} \frac{\varsigma^{n}-\varsigma^{n-1}}{\Delta t}\right\|^{2}+\left(d \frac{\varsigma^{n-1}-\widehat{\varsigma}^{n-1}}{\Delta t}, \frac{\varsigma^{n}-\varsigma^{n-1}}{\Delta t}\right) \\
& +\frac{\left\|\left(a^{n}\right)^{1 / 2}\left(\theta^{n}-\theta^{n-1}\right)\right\|^{2}}{2 \Delta t} \\
& +\frac{\left\|\left(a^{n}\right)^{1 / 2} \theta^{n}\right\|^{2}-\left\|\left(a^{n-1}\right)^{1 / 2} \theta^{n-1}\right\|^{2}}{2 \Delta t} \\
& -\left(\frac{a^{n}-a^{n-1}}{2 \Delta t} \theta^{n-1}, \theta^{n-1}\right)+\frac{\left\|\left(R^{n}\right)^{1 / 2}\left(\varsigma^{n}-\varsigma^{n-1}\right)\right\|^{2}}{2 \Delta t} \\
& +\frac{\left\|\left(R^{n}\right)^{1 / 2} \varsigma^{n}\right\|^{2}-\left\|\left(R^{n-1}\right)^{1 / 2} \varsigma^{n-1}\right\|^{2}}{2 \Delta t} \\
& \frac{\left(R^{n}-R^{n-1} \varsigma^{n-1}, \varsigma^{n-1}\right) \cdot}{2 \Delta t}
\end{aligned}
$$

Substitute (35) into (34) and multiply by $2 \Delta t$ to get

$$
\begin{aligned}
& \Delta t\left\|d^{1 / 2} \frac{\varsigma^{n}-\varsigma^{n-1}}{\Delta t}\right\|^{2}+\left\|\left(a^{n}\right)^{1 / 2}\left(\theta^{n}-\theta^{n-1}\right)\right\|^{2} \\
&+\left\|\left(R^{n}\right)^{1 / 2}\left(\varsigma^{n}-\varsigma^{n-1}\right)\right\|^{2} \\
&+\left(\left\|\left(a^{n}\right)^{1 / 2} \theta^{n}\right\|^{2}-\left\|\left(a^{n-1}\right)^{1 / 2} \theta^{n-1}\right\|^{2}\right) \\
&+\left(\left\|\left(R^{n}\right)^{1 / 2} \varsigma^{n}\right\|^{2}-\left\|\left(R^{n-1}\right)^{1 / 2} \varsigma^{n-1}\right\|^{2}\right) \\
& \leq C\left(d_{1}\right)(\Delta t)^{2} \int_{t_{n-1}}^{t_{n}}\left\|\frac{\partial^{2} u}{\partial \tau^{2}}\right\|^{2} d s \\
&+C\left(d_{1}\right) \int_{t_{n-1}}^{t_{n}}\left\|\frac{\partial \eta}{\partial \tau}\right\|^{2} d s+C\left(R_{1}\right) \Delta t\|\eta\|_{L^{\infty}\left(L^{2}\right)}^{2} \\
&-\Delta t\left(d \frac{\varsigma^{n-1}-\widehat{\varsigma}^{n-1}}{\Delta t}, \frac{\varsigma^{n}-\varsigma^{n-1}}{\Delta t}\right) \\
&+\Delta t\left(\frac{\int_{t_{n-1}}^{t_{n}} a_{t} d s}{2 \Delta t} \theta^{n-1}, \theta^{n-1}\right) \\
&+\Delta t R_{t}^{t_{n-1}} d s \\
& 2 \Delta t\left.\varsigma^{n-1}, \varsigma^{n-1}\right)
\end{aligned}
$$

Summing (36) from $n=1$ to $J$, the resulting inequality becomes

$$
\begin{aligned}
\Delta t \sum_{n=1}^{J} \| & d^{1 / 2} \frac{\varsigma^{n}-\varsigma^{n-1}}{\Delta t} \|^{2} \\
& +\sum_{n=1}^{J}\left(\left\|\left(a^{n}\right)^{1 / 2}\left(\theta^{n}-\theta^{n-1}\right)\right\|^{2}+\left\|\left(R^{n}\right)^{1 / 2}\left(\varsigma^{n}-\varsigma^{n-1}\right)\right\|^{2}\right) \\
& +\left\|\left(a^{J}\right)^{1 / 2} \theta^{J}\right\|^{2}+\left\|\left(R^{J}\right)^{1 / 2} \varsigma^{J}\right\|^{2} \\
\leq & \left\|\left(a^{0}\right)^{1 / 2} \theta^{0}\right\|^{2}+C\left(d_{1}\right)\left((\Delta t)^{2} \int_{t_{0}}^{t_{J}}\left\|\frac{\partial^{2} u}{\partial \tau^{2}}\right\|^{2} d s\right. \\
& +C\left(R_{1}\right)\|\eta\|_{L^{\infty}\left(L^{2}\right)}^{2}+C\left(\bar{a}_{1}\right) \Delta t \sum_{n=0}^{J-1}\left\|\theta^{n}\right\|^{2} \\
& \left.-\Delta t \sum_{n=1}^{t_{J}}\left\|\frac{\partial \eta}{\partial \tau}\right\|^{2} d s\right) \\
& +C\left(\bar{R}_{1}\right) \Delta t \sum_{n=0}^{J-1}\left\|\varsigma^{n}\right\|^{2} \\
& \\
& \\
& \\
& \\
\varsigma^{n-1}-\widehat{\varsigma}^{n-1} & \left.\frac{\varsigma^{n}-\varsigma^{n-1}}{\Delta t}\right)
\end{aligned}
$$

Using the same analysis as that in [29], we obtain

$$
\begin{aligned}
\Delta t & \sum_{n=1}^{J}\left(d \frac{\varsigma^{n-1}-\widehat{\varsigma}^{n-1}}{\Delta t}, \frac{\varsigma^{n}-\varsigma^{n-1}}{\Delta t}\right) \\
= & \Delta t \sum_{n=1}^{J}\left(d \frac{\varsigma^{n-1}-\widehat{\varsigma}^{n-1}}{\Delta t}, \frac{\varsigma^{n}}{\Delta t}\right) \\
& -\Delta t \sum_{n=1}^{J}\left(d \frac{\varsigma^{n-1}-\widehat{\varsigma}^{n-1}}{\Delta t}, \frac{\varsigma^{n-1}}{\Delta t}\right) \\
= & \Delta t \sum_{n=1}^{J}\left(d \frac{\varsigma^{n-1}-\varsigma^{n}-\widehat{\varsigma}^{n-1}+\widehat{\varsigma}^{n}}{\Delta t}, \frac{\varsigma^{n}}{\Delta t}\right) \\
& +\Delta t \sum_{n=1}^{J}\left(d \frac{\varsigma^{n}-\widehat{\varsigma}^{n}}{\Delta t}, \frac{\varsigma^{n}}{\Delta t}\right) \\
& -\Delta t \sum_{n=1}^{J}\left(d \frac{\varsigma^{n-1}-\widehat{\varsigma}^{n-1}}{\Delta t}, \frac{\varsigma^{n-1}}{\Delta t}\right) \\
= & \Delta t \sum_{n=1}^{J}\left(d \frac{\varsigma^{n-1}-\varsigma^{n}-\widehat{\varsigma}^{n-1}+\widehat{\varsigma}^{n}}{\Delta t}, \frac{\varsigma^{n}}{\Delta t}\right)+\left(d \frac{\varsigma^{J}-\widehat{\varsigma}^{J}}{\Delta t}, \varsigma^{J}\right)
\end{aligned}
$$




$$
\begin{aligned}
& \leq \Delta t \sum_{n=1}^{J}\left\|\frac{\varsigma^{n-1}-\varsigma^{n}-\widehat{\varsigma}^{n-1}+\widehat{\varsigma}^{n}}{\Delta t}\right\|\left\|\frac{d \varsigma^{n}}{\Delta t}\right\|+\left(d \frac{\varsigma^{J}-\widehat{\varsigma}^{J}}{\Delta t}, \varsigma^{J}\right) \\
& \leq \varepsilon \Delta t \sum_{n=1}^{J}\left\|\frac{\varsigma^{n-1}-\varsigma^{n}}{\Delta t}\right\|^{2}+C(\varepsilon) \Delta t \sum_{n=1}^{J}\left\|\varsigma^{n}\right\|^{2}+C\left(d_{1}\right)\left\|\varsigma^{J}\right\|^{2} .
\end{aligned}
$$

Substitute (31) and (38) into (37) to obtain

$$
\begin{aligned}
\left(d_{0}-\varepsilon\right) \Delta t \sum_{n=1}^{J}\left\|\frac{\varsigma^{n}-\varsigma^{n-1}}{\Delta t}\right\|^{2} & \\
& +\sum_{n=1}^{J}\left(\left\|\left(a^{n}\right)^{1 / 2}\left(\theta^{n}-\theta^{n-1}\right)\right\|^{2}+\left\|\left(R^{n}\right)^{1 / 2}\left(\varsigma^{n}-\varsigma^{n-1}\right)\right\|^{2}\right) \\
& +\left\|\left(a^{J}\right)^{1 / 2} \theta^{J}\right\|^{2}+\left\|\left(R^{J}\right)^{1 / 2} \varsigma^{J}\right\|^{2} \\
\leq & C\left(a_{0}, d_{1}, R_{1}\right)\left((\Delta t)^{2} \int_{t_{0}}^{t_{J}}\left\|\frac{\partial^{2} u}{\partial \tau^{2}}\right\|^{2} d s+\int_{t_{0}}^{t_{J}}\left\|\frac{\partial \eta}{\partial \tau}\right\|^{2} d s\right) \\
& +C\left(a_{0}, d_{1}, R_{1}, \bar{R}_{1}\right)\|\eta\|_{L^{\infty}}^{2}\left(L^{2}\right)+C\left(\bar{a}_{1}\right) \Delta t \sum_{n=0}^{J-1}\left\|\theta^{n}\right\|^{2} .
\end{aligned}
$$

Using Gronwall lemma, we have

$$
\begin{aligned}
\Delta t \sum_{n=1}^{J}\left\|\frac{\varsigma^{n}-\varsigma^{n-1}}{\Delta t}\right\|^{2}+\left\|\theta^{J}\right\|^{2}+\left\|\left(R^{J}\right)^{1 / 2} \varsigma^{J}\right\|^{2} \\
\leq C\left(a_{0}, d_{1}, R_{1}\right)\left((\Delta t)^{2} \int_{t_{0}}^{t_{J}}\left\|\frac{\partial^{2} u}{\partial \tau^{2}}\right\|^{2} d s+\int_{t_{0}}^{t_{J}}\left\|\frac{\partial \eta}{\partial \tau}\right\|^{2} d s\right) \\
+C\left(a_{0}, d_{1}, R_{1}, \bar{R}_{1}\right)\|\eta\|_{L^{\infty}\left(L^{2}\right)}^{2} .
\end{aligned}
$$

Taking $\mathbf{z}_{h}=\xi^{n}$ in (21a), (21b), and (21c), we get

$$
\left\|\xi^{n}\right\|^{2} \leq C\left\|\theta^{n}\right\|^{2}
$$

Substitute (40) into (41) to get

$$
\begin{aligned}
\left\|\xi^{J}\right\|^{2} \leq & C\left(a_{0}, d_{1}, R_{1}\right)\left((\Delta t)^{2} \int_{t_{0}}^{t_{J}}\left\|\frac{\partial^{2} u}{\partial \tau^{2}}\right\|^{2} d s+\int_{t_{0}}^{t_{J}}\left\|\frac{\partial \eta}{\partial \tau}\right\|^{2} d s\right) \\
& +C\left(a_{0}, d_{1}, R_{1}, \bar{R}_{1}\right)\|\eta\|_{L^{\infty}\left(L^{2}\right)}^{2}
\end{aligned}
$$

Taking $\mathbf{w}_{h}=\nabla \varsigma^{n}$ in (21b), we have

$\left\|\nabla \varsigma^{n}\right\|^{2} \leq\left\|\theta^{n}\right\|^{2}$

$$
\begin{aligned}
\leq & C\left(a_{0}, d_{1}, R_{1}\right)\left((\Delta t)^{2} \int_{t_{0}}^{t_{J}}\left\|\frac{\partial^{2} u}{\partial \tau^{2}}\right\|^{2} d s+\int_{t_{0}}^{t_{J}}\left\|\frac{\partial \eta}{\partial \tau}\right\|^{2} d s\right) \\
& +C\left(a_{0}, d_{1}, R_{1}, \bar{R}_{1}\right)\|\eta\|_{L^{\infty}\left(L^{2}\right)}^{2}
\end{aligned}
$$

Combining (14)-(16), (31), (40), (43), (42), and the triangle inequality, we complete the proof.

Remark 9. Compared to the results in [29], we can obtain the optimal a priori error estimates in $H^{1}$-norm for the scalar unknown $u$ in Theorem 8 .

\section{Numerical Experiment}

In this section, in order to confirm our theoretical results for the novel characteristic expanded mixed finite element method, we consider the following test problem:

$$
\begin{gathered}
d(\mathbf{x}) \frac{\partial u}{\partial t}+\mathbf{c}(\mathbf{x}, t) \cdot \nabla u-\nabla \cdot(a(\mathbf{x}, t) \nabla u)+R(\mathbf{x}, t) u \\
=f(\mathbf{x}, t), \quad(\mathbf{x}, t) \in \Omega \times J
\end{gathered}
$$

with boundary condition

$$
u(\mathbf{x}, t)=0, \quad(\mathbf{x}, t) \in \partial \Omega \times \bar{J},
$$

and initial condition

$$
u(\mathbf{x}, 0)=x_{1} x_{2}\left(x_{1}-1\right)\left(x_{2}-1\right)\left(2 x_{2}-1\right), \quad \mathbf{x} \in \Omega,
$$

where $\Omega=[0,1] \times[0,1], \mathbf{x}=\left(x_{1}, x_{2}\right), J=(0,1], a(\mathbf{x}, t)=$ $1+2 x_{1}^{2}+x_{2}^{2}, \mathbf{c}(\mathbf{x}, t)=\left(1+x_{1}^{2}+x_{2}^{2}+t^{2}, 1+x_{1}^{2}+x_{2}^{2}+t^{2}\right)$, $R(\mathbf{x}, t)=1+x_{1}^{2}+2 x_{2}^{2}$, and $d(\mathbf{x})=1$ and $f(\mathbf{x}, t)$ is chosen so that the exact solution for the scalar unknown function is

$$
u(\mathbf{x}, t)=e^{-t} x_{1} x_{2}\left(x_{1}-1\right)\left(x_{2}-1\right)\left(2 x_{2}-1\right) .
$$

The corresponding exact gradient function is

$$
\lambda=\nabla u=e^{-t}\left[\begin{array}{c}
x_{2}\left(2 x_{1}-1\right)\left(x_{2}-1\right)\left(2 x_{2}-1\right) \\
x_{1}\left(x_{1}-1\right)\left(6 x_{2}^{2}-6 x_{2}+1\right)
\end{array}\right],
$$

and its exact flux function is

$$
\begin{aligned}
\sigma & =-a \nabla u \\
& =-e^{-t}\left(1+2 x_{1}^{2}+x_{2}^{2}\right)\left[\begin{array}{c}
x_{2}\left(2 x_{1}-1\right)\left(x_{2}-1\right)\left(2 x_{2}-1\right) \\
x_{1}\left(x_{1}-1\right)\left(6 x_{2}^{2}-6 x_{2}+1\right)
\end{array}\right] .
\end{aligned}
$$

We divide the domain $\Omega=[0,1] \times[0,1]$ into the triangulations of spatial mesh parameter $h$ uniformly and use the backward Euler procedure with uniform time discretization parameter $\Delta t$. We consider the piecewise linear space $V_{h}$ with index $k=1$ and the corresponding piecewise constant space $\mathbf{W}_{h}$ with index $k=0$.

In Table 1, we get the optimal a priori error estimate in $L^{2}$-norm for the scalar unknown $u$ with $h=2 \sqrt{2} \Delta t=$ $\sqrt{2} / 16, \sqrt{2} / 32, \sqrt{2} / 64$. At the same time, we also obtain the optimal a priori error estimate in $H^{1}$-norm for the scalar unknown $u$.

In Table 2, we obtain some convergence results in $\left(L^{2}(\Omega)\right)^{2}$-norm for the gradient $\lambda$ with $h=2 \sqrt{2} \Delta t=\sqrt{2} / 16$, $\sqrt{2} / 32, \sqrt{2} / 64$ in Table 2 . The similar results are obtained for the flux $\sigma$ in Table 2. From the data obtained in Table 2, we can 
TABLE 1: The errors and convergence rate for $u$.

\begin{tabular}{|c|c|c|c|c|}
\hline$(h, \Delta t)$ & $\left\|u-u_{h}\right\|_{L^{\infty}\left(L^{2}(\Omega)\right)}$ & Rate & $\left\|u-u_{h}\right\|_{L^{\infty}\left(H^{1}(\Omega)\right)}$ & Rate \\
\hline$(\sqrt{2} / 8,1 / 16)$ & $1.3622 e-003$ & & $2.6286 e-002$ & \\
\hline$(\sqrt{2} / 16,1 / 32)$ & $4.1958 e-004$ & 1.6989 & $1.3583 e-002$ & 0.9525 \\
\hline$(\sqrt{2} / 32,1 / 64)$ & $1.3172 e-004$ & 1.6715 & $6.8843 e-003$ & 0.9804 \\
\hline
\end{tabular}

TABLE 2: The errors and convergence rate for $\lambda$ and $\sigma$.

\begin{tabular}{|c|c|c|c|c|}
\hline$(h, \Delta t)$ & $\left\|\lambda-\lambda_{h}\right\|_{L^{\infty}\left(\left(L^{2}(\Omega)\right)^{2}\right)}$ & Rate & $\left\|\sigma-\sigma_{h}\right\|_{L^{\infty}\left(\left(L^{2}(\Omega)\right)^{2}\right)}$ & Rate \\
\hline$(\sqrt{2} / 8,1 / 16)$ & $2.6251 e-002$ & & $5.4313 e-002$ & \\
\hline$(\sqrt{2} / 16,1 / 32)$ & $1.3576 e-002$ & 0.9513 & $2.9081 e-002$ & 0.9013 \\
\hline$(\sqrt{2} / 32,1 / 64)$ & $6.8830 e-003$ & 0.9800 & $1.5018 e-002$ & 0.9534 \\
\hline
\end{tabular}

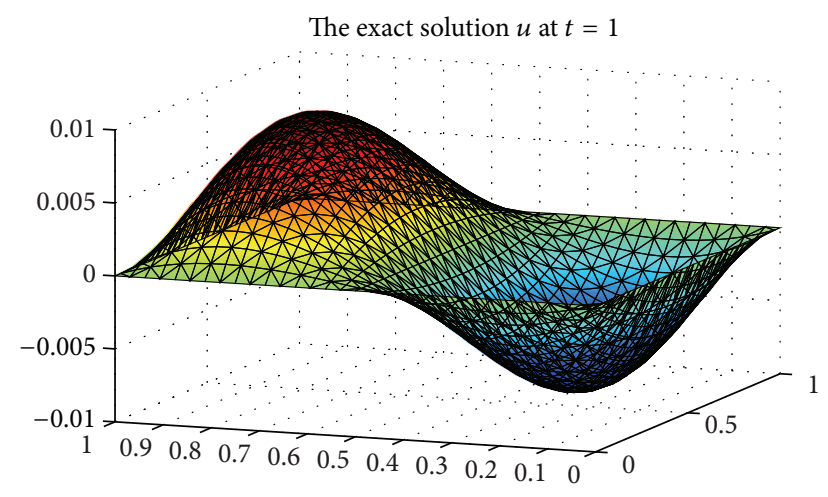

FIGURE 1: The surface for the exact solution $u$.

find that the numerical results confirm the theoretical results of Theorem 8 .

Figure 1 shows the surface for the exact solution $u$ at $t=$ 1 , and Figure 2 describes the corresponding surface for the numerical solution $u_{h}$ with $h=2 \sqrt{2} \Delta t=\sqrt{2} / 16$ at $t=1$. In Figures 1 and 2, we can find easily that the exact solution $u$ is approximated very well by the numerical solution $u_{h}$.

Figures 3 and 4 show the surface of the exact gradient function $\lambda$ and the surface of the numerical gradient function $\lambda_{h}$ with $h=2 \sqrt{2} \Delta t=\sqrt{2} / 16$ at $t$, respectively. A similar comparison between the surface of the exact flux function $\sigma$ and the surface of the numerical flux function $\sigma_{h}$ in Figures 5 and 6 also is made.

From the convergence results for $\left\|u-u_{h}\right\|_{L^{\infty}\left(L^{2}(\Omega)\right)}, \| u-$ $u_{h}\left\|_{L^{\infty}\left(H^{1}(\Omega)\right)},\right\| \lambda-\lambda_{h} \|_{L^{\infty}\left(\left(L^{2}(\Omega)\right)^{2}\right)}$, and $\left\|\sigma-\sigma_{h}\right\|_{L^{\infty}\left(\left(L^{2}(\Omega)\right)^{2}\right)}$ in Tables 1 and 2 and Figures 1 and 2, we find that the numerical results confirm our theoretical analysis.

\section{Concluding Remarks}

In this paper, we propose and study a novel characteristic expanded mixed finite element method, which combines the novel expanded mixed method [24] applied to approximating the diffusion term and the characteristic method that handled the hyperbolic part, for reaction-convection-diffusion equations. Compared to Chen's expanded mixed method, the

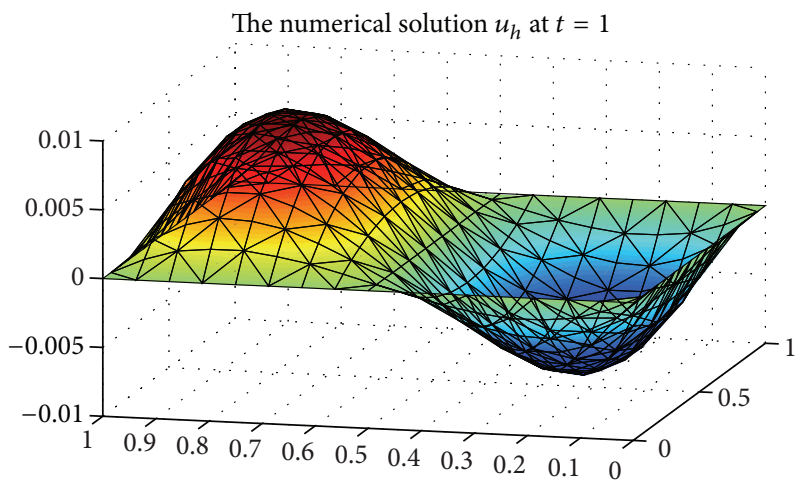

FIGURE 2: The surface for the numerical solution $u_{h}$.

gradient for our method belongs to the square integrable space instead of the classical $\mathbf{H}(\operatorname{div} ; \Omega)$ space. We derive a priori error estimates based on backward Euler method. Moreover, we prove the optimal a priori error estimates in $L^{2}$ - and $H^{1}$-norms for the scalar unknown $u$ and a priori error estimates in $\left(L^{2}\right)^{2}$-norm for its gradient $\lambda$ and its flux $\sigma$. Finally, we choose a test problem to confirm our theoretical results. In the near future, the proposed characteristic expanded mixed scheme will be applied to other linear/nonlinear evolution equations, such as nonlinear reaction-diffusion equations, linear/nonlinear convection-dominated Sobolev equations, and time-dependent convection-diffusion optimal control problems.

\section{Acknowledgments}

The authors thank the anonymous referees and editors for their helpful comments and suggestions, which greatly improve the paper. This work is supported by the National Natural Science Fund (11061021), Natural Science Fund of Inner Mongolia Autonomous Region (2012MS0108, 2012MS0106, and 2011BS0102), Scientific Research Projection of Higher Schools of Inner Mongolia (NJZZ12011, NJ10006, NJ10016, and NJZY13199), Key Project of Chinese Ministry of Education (12024), and the Program of Higher-Level 
The exact solution $\lambda_{1}$ at $t=1$

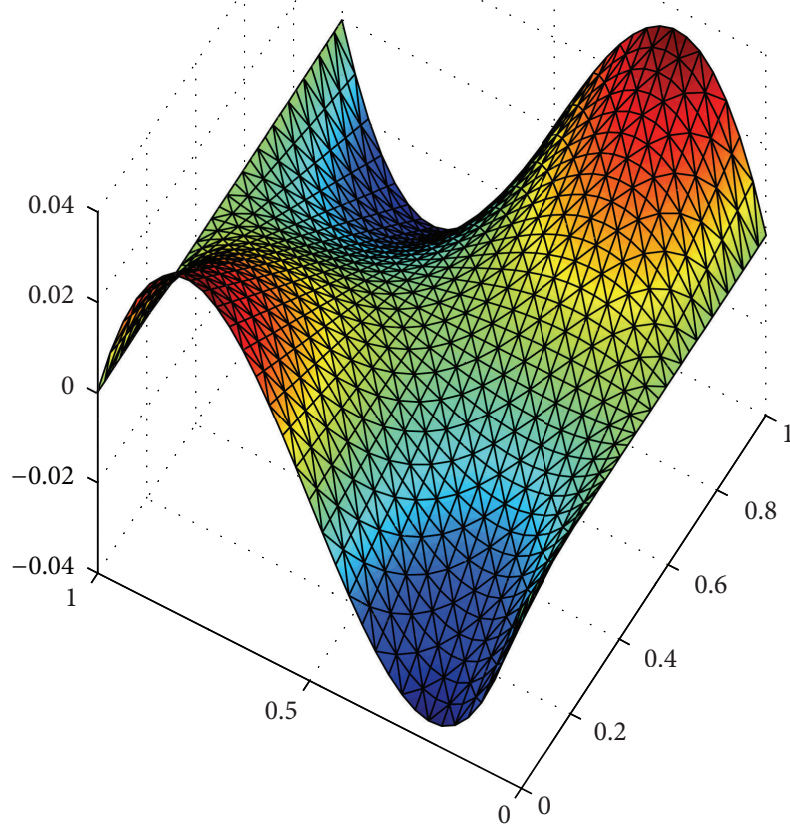

(a)
The exact solution $\lambda_{2}$ at $t=1$

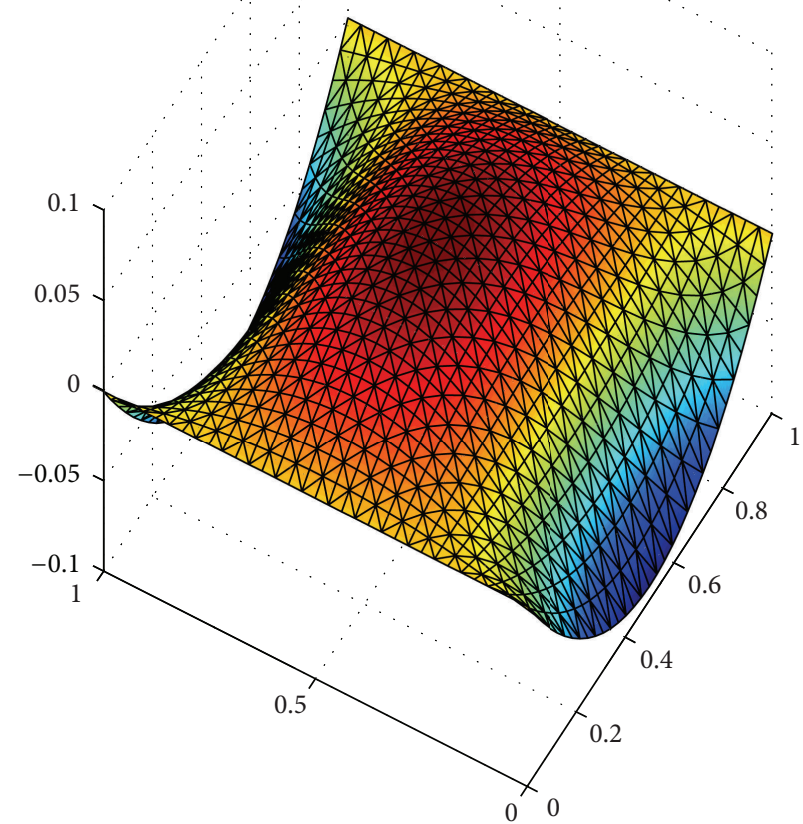

(b)

Figure 3: The surface for the exact solution $\lambda=\left(\lambda_{1}, \lambda_{2}\right)$.

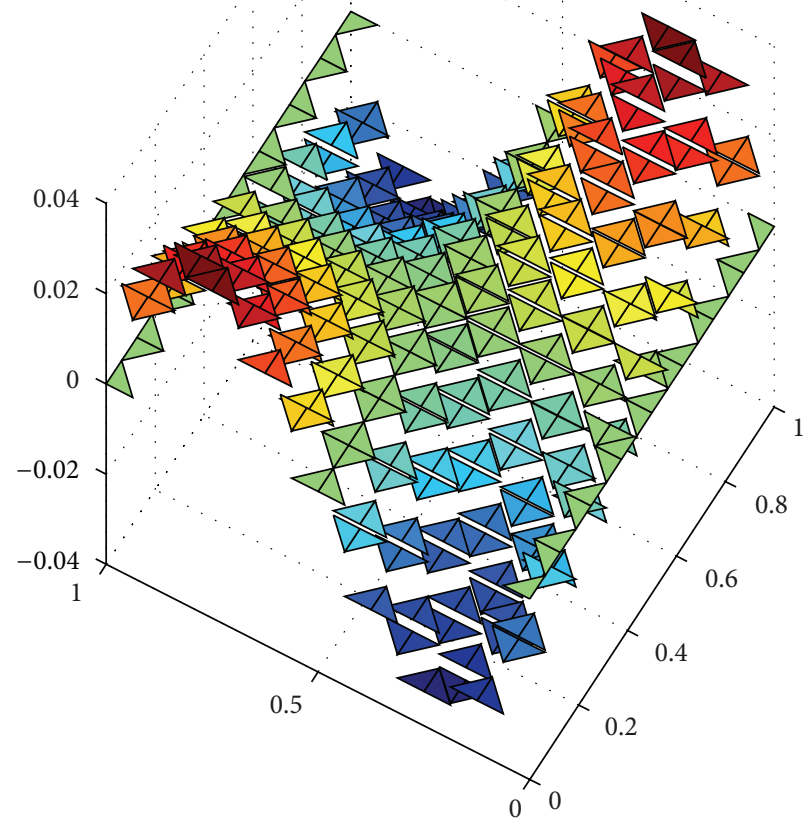

(a)

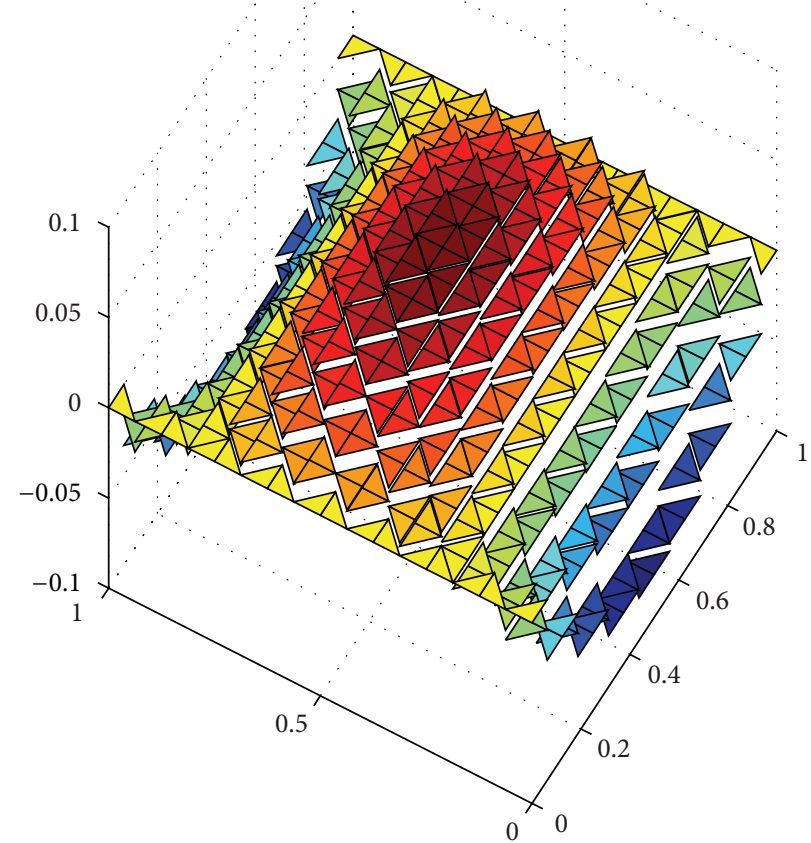

(b)

FIgure 4: The surface for the numerical solution $\lambda_{h}=\left(\lambda_{1 h}, \lambda_{2 h}\right)$. 
The exact solution $\sigma_{1}$ at $t=1$

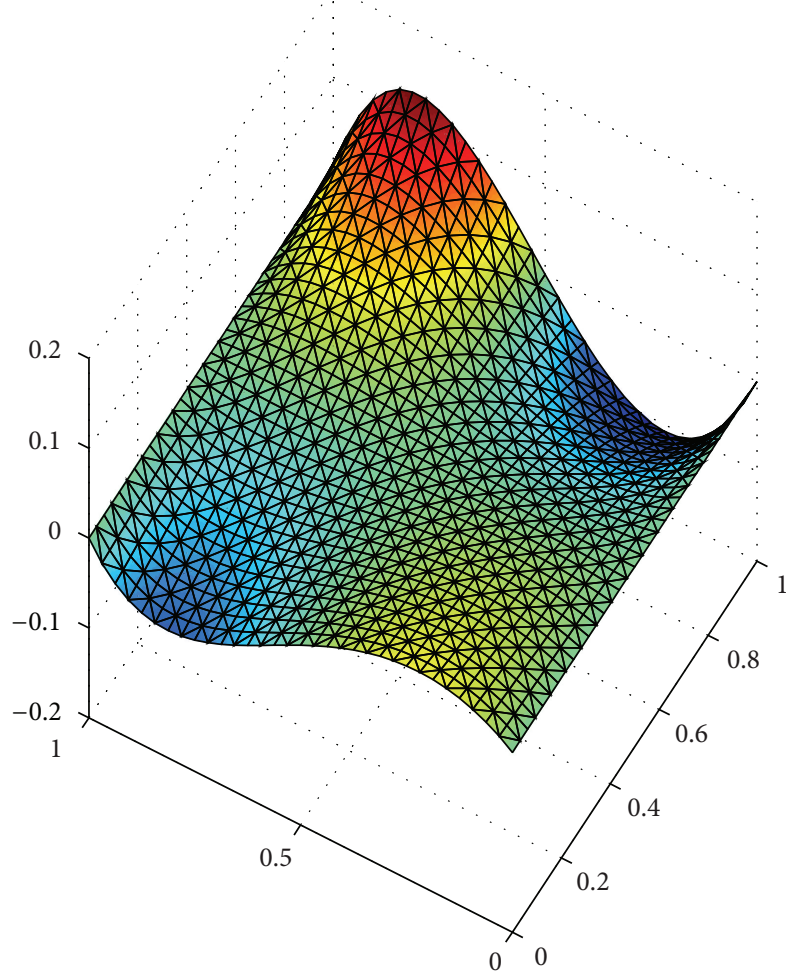

(a)
The exact solution $\sigma_{2}$ at $t=1$

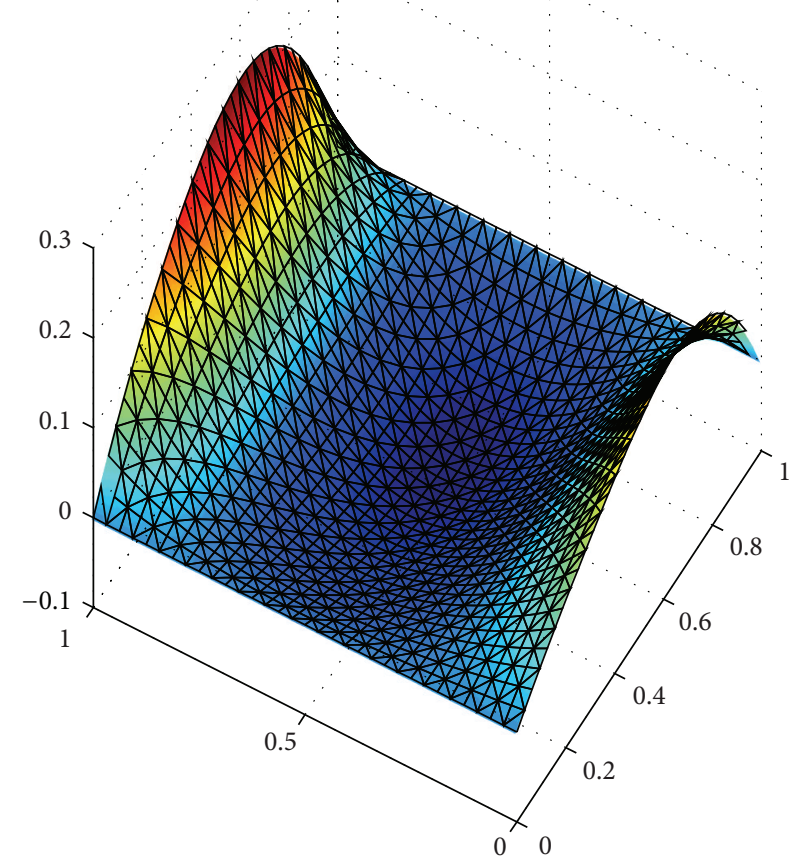

(b)

FIgURE 5: The surface for the exact solution $\sigma=\left(\sigma_{1}, \sigma_{2}\right)$.

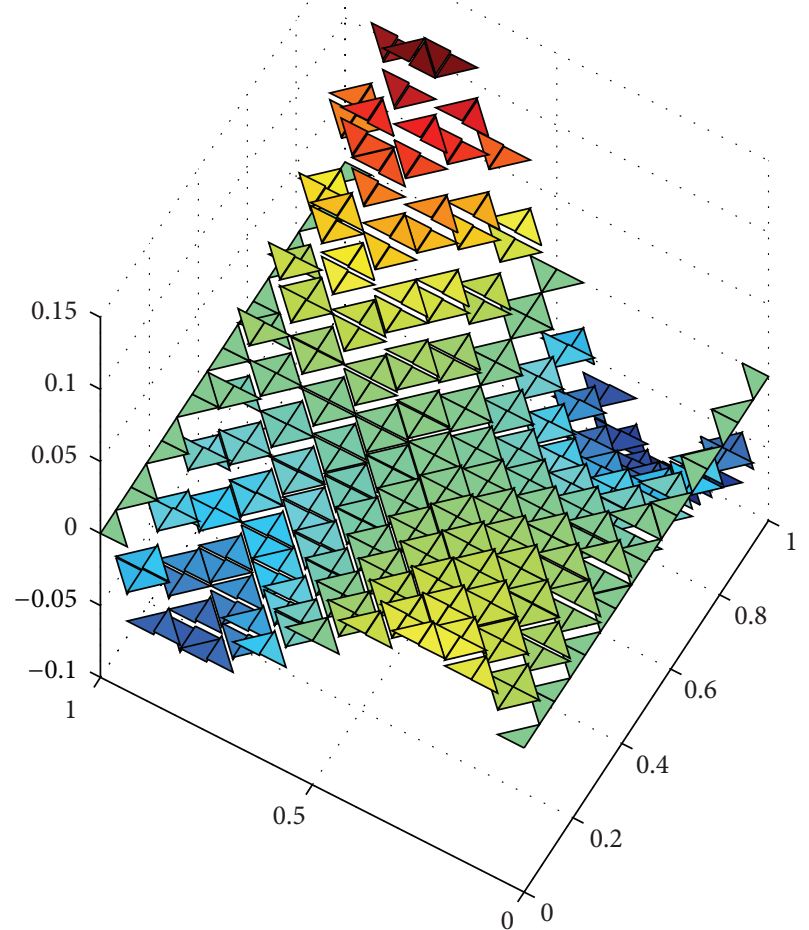

(a)

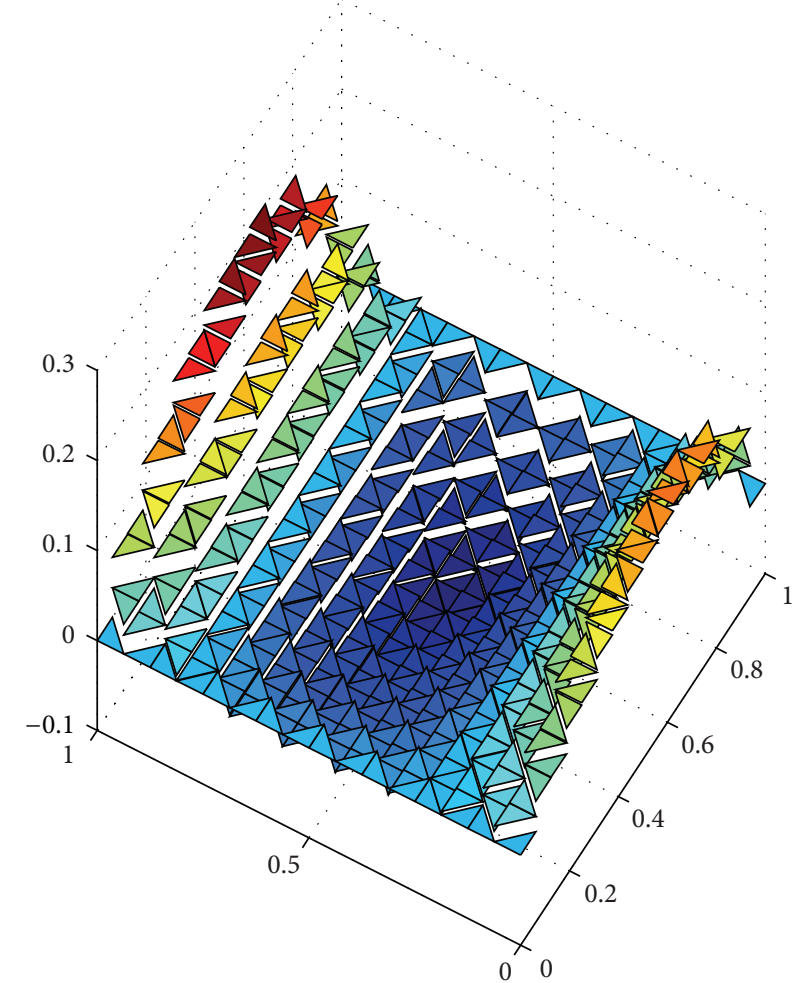

(b)

FIgURE 6: The surface for the numerical solution $\sigma_{h}=\left(\sigma_{1 h}, \sigma_{2 h}\right)$. 
Talents of Inner Mongolia University (125119, Z200901004, and 30105-125132).

\section{References}

[1] C. Johnson and V. Thomée, "Error estimates for some mixed finite element methods for parabolic type problems," RAIRO Analyse Numérique, vol. 15, no. 1, pp. 41-78, 1981.

[2] C. S. Woodward and C. N. Dawson, "Analysis of expanded mixed finite element methods for a nonlinear parabolic equation modeling flow into variably saturated porous media," SIAM Journal on Numerical Analysis, vol. 37, no. 3, pp. 701-724, 2000.

[3] H. Z. Chen and H. Wang, "An optimal-order error estimate on an $H^{1}$-Galerkin mixed method for a nonlinear parabolic equation in porous medium flow," Numerical Methods for Partial Differential Equations, vol. 26, no. 1, pp. 188-205, 2010.

[4] Z. D. Luo, Z. H. Xie, Y. Q. Shang, and J. Chen, "A reduced finite volume element formulation and numerical simulations based on POD for parabolic problems," Journal of Computational and Applied Mathematics, vol. 235, no. 8, pp. 2098-2111, 2011.

[5] P. Sun, Z. Luo, and Y. Zhou, "Some reduced finite difference schemes based on a proper orthogonal decomposition technique for parabolic equations," Applied Numerical Mathematics, vol. 60, no. 1-2, pp. 154-164, 2010.

[6] Z. X. Chen, S. H. Chou, and D. Y. Kwak, "Characteristicmixed covolume methods for advection-dominated diffusion problems," Numerical Linear Algebra with Applications, vol. 13, no. 9, pp. 677-697, 2006.

[7] S. He, H. Li, Y. Liu, Z. C. Fang et al., "A splitting mixed spacetime discontinuous Galerkin method for parabolic problems," Procedia Engineering, vol. 31, pp. 1050-1059, 2012.

[8] Y. Liu, H. Li, and S. He, "Mixed time discontinuous spacetime finite element method for convection diffusion equations," Applied Mathematics and Mechanics, vol. 29, no. 12, pp. 15791586, 2008.

[9] D. P. Yang, "Least-squares mixed finite element methods for nonlinear parabolic problems," Journal of Computational Mathematics, vol. 20, no. 2, pp. 153-164, 2002.

[10] H. X. Rui, S. D. Kim, and S. Kim, "Split least-squares finite element methods for linear and nonlinear parabolic problems," Journal of Computational and Applied Mathematics, vol. 223, no. 2, pp. 938-952, 2009.

[11] D. Shi and B. Y. Zhang, "Nonconforming finite element method for nonlinear parabolic equations," Journal of Systems Science \& Complexity, vol. 23, no. 2, pp. 395-402, 2010.

[12] J. C. Li, "Optimal uniform convergence analysis for a twodimensional parabolic problem with two small parameters," International Journal of Numerical Analysis and Modeling, vol. 2, no. 1, pp. 107-126, 2005.

[13] Y. Chen, Y. Huang, and D. Yu, "A two-grid method for expanded mixed finite-element solution of semilinear reaction-diffusion equations," International Journal for Numerical Methods in Engineering, vol. 57, no. 2, pp. 193-209, 2003.

[14] Y. Chen, H. W. Liu, and S. Liu, "Analysis of two-grid methods for reaction-diffusion equations by expanded mixed finite element methods," International Journal for Numerical Methods in Engineering, vol. 69, no. 2, pp. 408-422, 2007.

[15] Y. Chen, P. Luan, and Z. Lu, "Analysis of two-grid methods for nonlinear parabolic equations by expanded mixed finite element methods," Advances in Applied Mathematics and Mechanics, vol. 1, no. 6, pp. 830-844, 2009.
[16] W. Liu, H. X. Rui, and H. Guo, "A two-grid method with expanded mixed element for nonlinear reaction-diffusion equations," Acta Mathematicae Applicatae Sinica, vol. 27, no. 3, pp. 495-502, 2011.

[17] Z. X. Chen, Expanded Mixed Finite Element Methods For Linear Second-Order Elliptic Problems I, vol. 1219 of IMA preprint series, Institute for Mathematics and its Applications, University of Minnesota, Minneapolis, Minn, USA, 1994.

[18] Z. Chen, "Expanded mixed finite element methods for linear second-order elliptic problems. I," RAIRO Modélisation Mathématique et Analyse Numérique, vol. 32, no. 4, pp. 479-499, 1998.

[19] T. Arbogast, M. F. Wheeler, and I. Yotov, "Mixed finite elements for elliptic problems with tensor coefficients as cell-centered finite differences," SIAM Journal on Numerical Analysis, vol. 34, no. 2, pp. 828-852, 1997.

[20] Z. Chen, "Expanded mixed finite element methods for quasilinear second order elliptic problems. II," RAIRO Modélisation Mathématique et Analyse Numérique, vol. 32, no. 4, pp. 501-520, 1998.

[21] H. Rui and T. Lu, "An expanded mixed covolume method for elliptic problems," Numerical Methods for Partial Differential Equations, vol. 21, no. 1, pp. 8-23, 2005.

[22] D. Kim and E. J. Park, "A posteriori error estimator for expanded mixed hybrid methods," Numerical Methods for Partial Differential Equations, vol. 23, no. 2, pp. 330-349, 2007.

[23] Y. Liu, H. Li, J. Wang, and W. Gao, "A new positive definite expanded mixed finite element method for parabolic integrodifferential equations," Journal of Applied Mathematics, vol. 2012, Article ID 391372, 24 pages, 2012.

[24] Y. Liu, H. Li et al., "A new expanded mixed finite element method for elliptic equations," http://nmg.zsksedu.com/3267/ 2012011690642.htm.

[25] S. C. Chen and H. R. Chen, "New mixed element schemes for a second-order elliptic problem," Mathematica Numerica Sinica, vol. 32, no. 2, pp. 213-218, 2010.

[26] F. Shi, J. P. Yu, and K. T. Li, "A new stabilized mixed finiteelement method for Poisson equation based on two local Gauss integrations for linear element pair," International Journal of Computer Mathematics, vol. 88, no. 11, pp. 2293-2305, 2011.

[27] D. Y. Shi and Y. D. Zhang, "High accuracy analysis of a new nonconforming mixed finite element scheme for Sobolev equations," Applied Mathematics and Computation, vol. 218, no. 7, pp. 3176-3186, 2011.

[28] P. G. Ciarlet, The Finite Element Method for Elliptic Problems, North-Holland, Amsterdam, The Netherlands, 1978.

[29] L. Guo and H. Z. Chen, "An expanded characteristic-mixed finite element method for a convection-dominated transport problem," Journal of Computational Mathematics, vol. 23, no. 5, pp. 479-490, 2005.

[30] L. Li, P. Sun, and Z. D. Luo, "A new mixed finite element formulation and error estimates for parabolic problems," Acta Mathematica Scientia, vol. 32A, no. 6, pp. 1158-1165, 2012.

[31] J. Douglas, Jr. and T. F. Russell, "Numerical methods for convection-dominated diffusion problems based on combining the method of characteristics with finite element or finite difference procedures," SIAM Journal on Numerical Analysis, vol. 19, no. 5, pp. 871-885, 1982. 


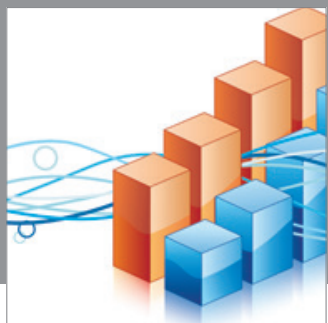

Advances in

Operations Research

mansans

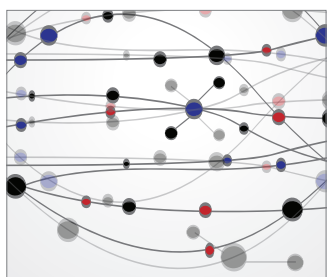

The Scientific World Journal
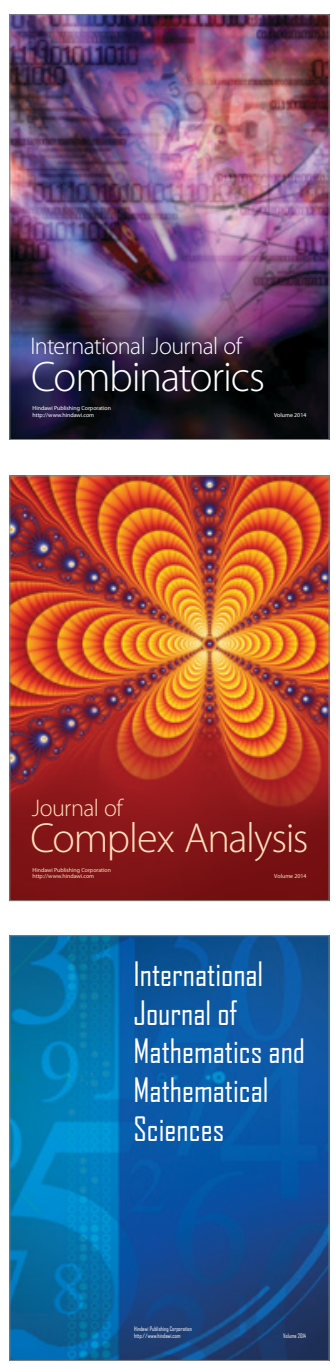
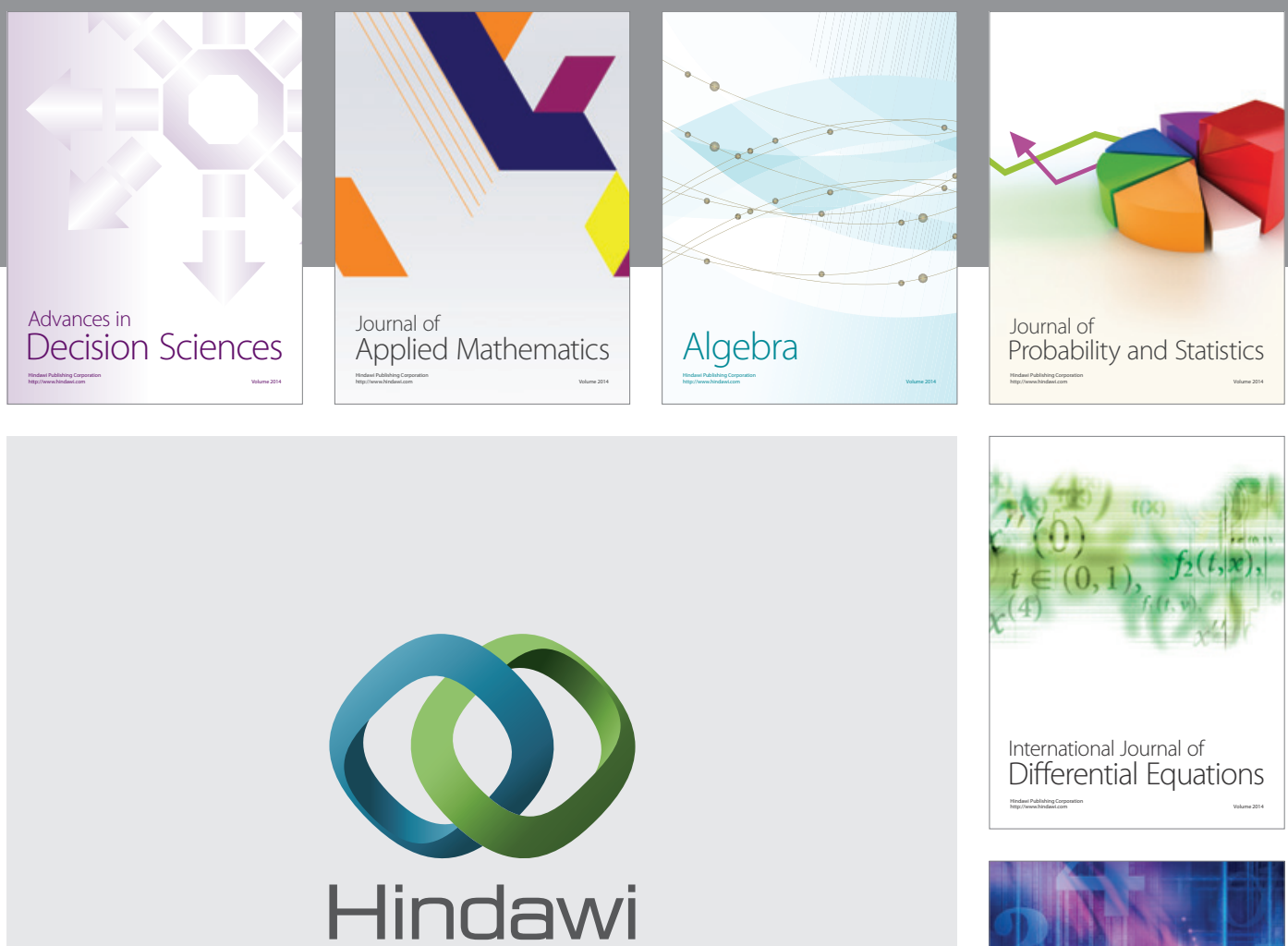

Submit your manuscripts at http://www.hindawi.com
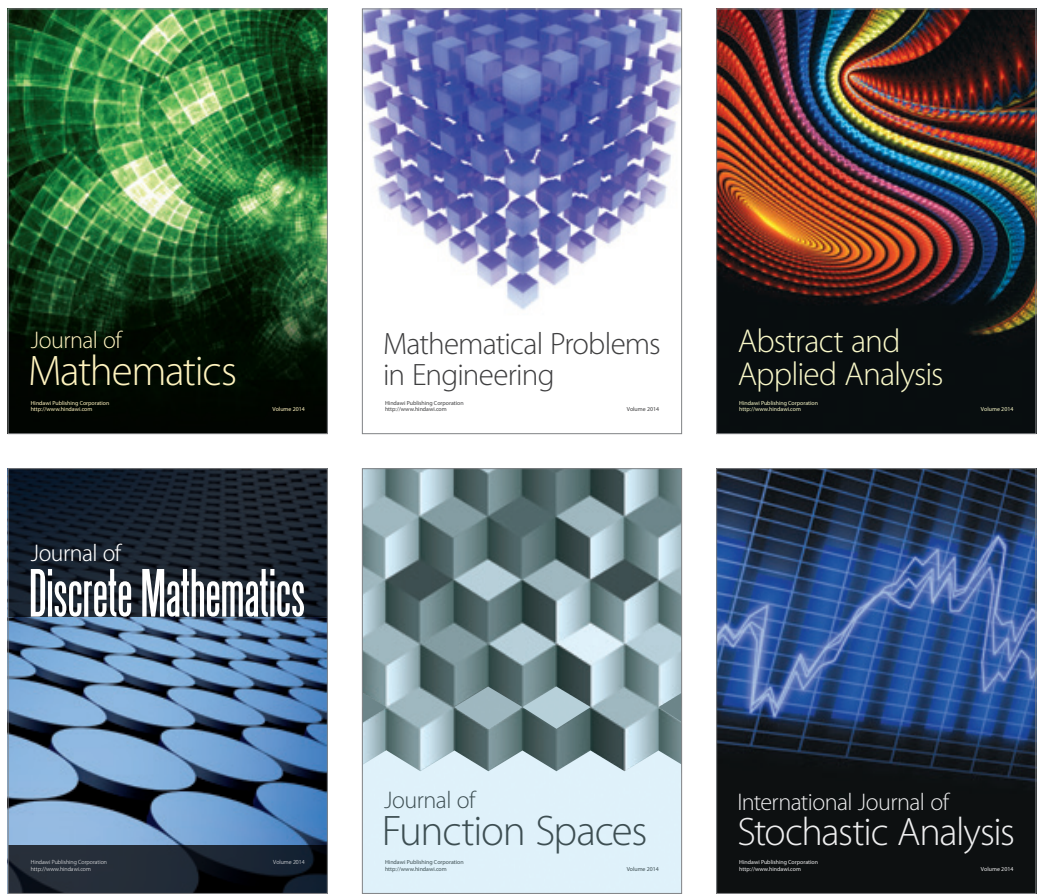

Journal of

Function Spaces

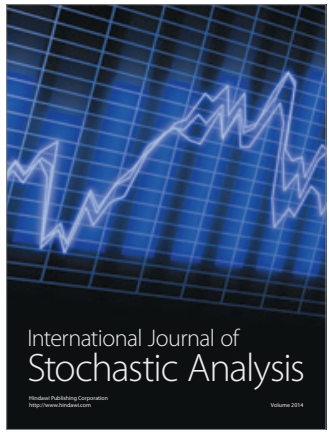

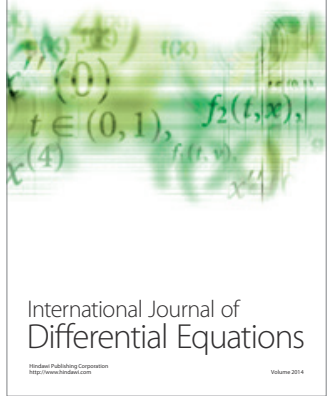
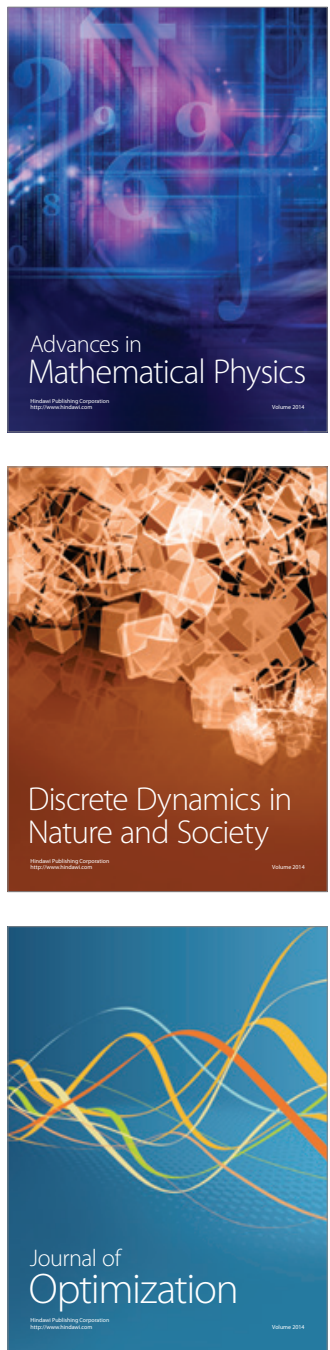